This item was submitted to Loughborough's Research Repository by the author.

Items in Figshare are protected by copyright, with all rights reserved, unless otherwise indicated.

\title{
Optimised sensor configurations for a Maglev suspension
}

PLEASE CITE THE PUBLISHED VERSION

PUBLISHER

(C) The International Federation of Automatic Control (IFAC)

LICENCE

CC BY-NC-ND 4.0

\section{REPOSITORY RECORD}

Michail, Konstantinos, Argyrios C. Zolotas, and Roger M. Goodall. 2019. "Optimised Sensor Configurations for a Maglev Suspension". figshare. https://hdl.handle.net/2134/3351. 
This item was submitted to Loughborough's Institutional Repository by the author and is made available under the following Creative Commons Licence conditions.

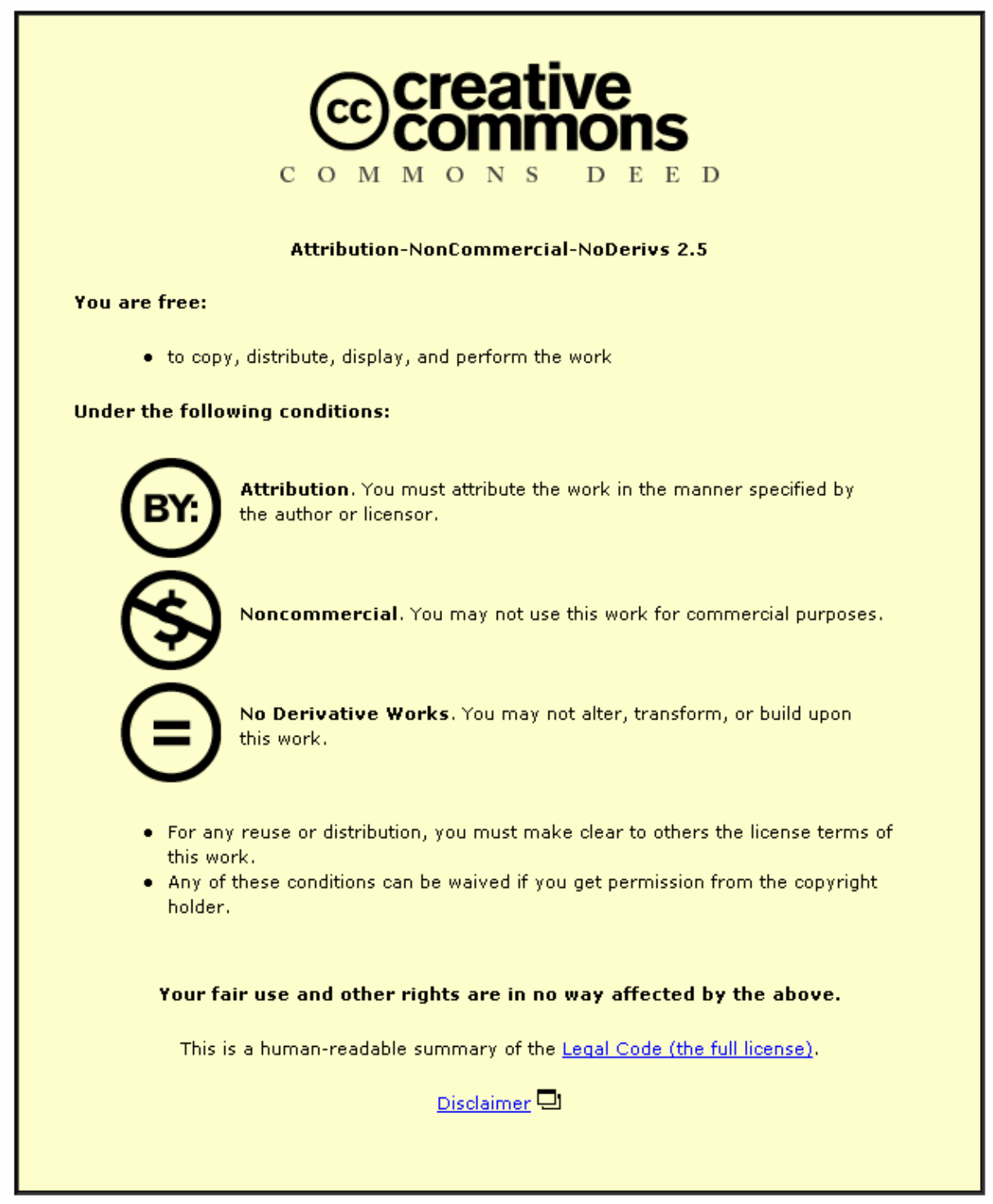

For the full text of this licence, please go to: http://creativecommons.org/licenses/by-nc-nd/2.5/ 


\title{
Optimised sensor configurations for a Maglev suspension
}

\author{
K.Michail, A.C. Zolotas, R.M. Goodall \\ Department of Electronic and Electrical Engineering, Loughborough \\ University, UK, \\ e-mail:\{k.michail,a.c.zolotas,r.m.goodall\}@lboro.ac.uk.
}

\begin{abstract}
This paper discusses a systematic approach for selecting the minimum number of sensors for an Electromagnetic levitation system that satisfies both deterministic and stochastic performance objectives. The controller tuning is based upon the utilisation of a recently developed genetic algorithm, namely NSGAII. Two controller structures are discussed, an inner loop classical solution for illustrating the efficacy of the NSGAII tuning and a Linear quadratic gaussian structure particularly on sensor optimization.
\end{abstract}

\section{INTRODUCTION}

In recent years, MAGnetic LEVitation (MAGLEV) systems have been attractive to transport industry due to a number of advantages they offer over the conventional wheel-on-rail systems. In particular, maglev trains have no mechanical contacts with the rail and thus maintenance costs are reduced, although in general building maglev rail infrastructure is more expensive than conventional rail infrastructure. An interesting survey can be found in Lee (2006).The work presented in this paper is a system study on sensor optimization for a quarter car magnetic suspension model, which aims to satisfy both disturbance rejection and robustness to parametric changes as well as the best ride quality with the minimum possible effort based on the minimum possible sensor set configuration. In fact, the problem is posed in a multiobjective optimization framework to optimize the controller parameters, via a heuristic algorithm, for each sensor set. Evolutionary algorithms are widely used in control engineering and have proved to be very efficient for controller optimization in a number of problems (see Fleming (2002)). Numerous genetic algorithms have been developed (see Abdullah (2006)), while for the purposes of this work a recently developed genetic algorithm by Deb (2002) NSGA-II, based on non-dominated sorting of population, is utilised for tuning the parameters of all controllers presented. In particular, the efficacy of NSGAII tuning is illustrated on a classical structure with inner-loop, while a Linear quadratic gaussian (LQG) structure is further utilized for sensor selection.

The paper is organized as follows. The linear time invariant model of a quarter car is presented in section 2 along with description of the deterministic and stochastic inputs. Section 3 presents both the classical control approach and the LQG technique applied for each sensor set. Section 4 discusses the simulation results, while conclusions are drawn in section 5 .

\section{MODEL DESCRIPTION}

The diagram of a one degree-of-freedom, 'quarter-car' electromagnetic suspension system is shown in Fig.1. The sus- pension consists of an electromagnet with a ferromagnetic core and a coil of $N$ turns which is attracted to the rail that is made out of ferromagnetic material. The carriage mass is attached on the electromagnet, with $z_{t}$ the rail position and $z$ the electromagnet position. The air gap $\left(z_{t}-z\right)$ is to be maintained close to the operating condition required. The LTI state space model is derived by considering the

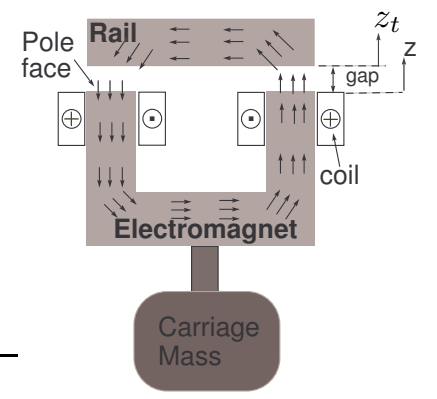

Fig. 1. Suspension system for MAGLEV

operating point (nominal) values of the coil current $I_{0}$, flux $B_{0}$, force $F_{0}$ and air gap $G_{0}$. The following relationships hold

$$
\begin{array}{ll}
F=f+F_{0}, & B=b+B_{0} \\
G=\left(z_{t}-z\right)+G_{0}, I=i+I_{0}
\end{array}
$$

where, $f, b,\left(z_{t}-z\right)$ and $i$ are small variations around their nominal values. The fundamental magnetic relationships are $F \propto B^{2}$ and $B \propto I / G$, thus, the linearized expressions for the magnet are (see Goodall (1985) for derivation)

$$
\begin{array}{r}
b=K_{i} i-K_{\left(z_{t}-z\right)}\left(z_{t}-z\right) \\
f=K_{b} b
\end{array}
$$

where $K_{i}=B_{0} / I_{0}, K_{\left(z_{t}-z\right)}=B_{0} / G_{0}$ and $K_{b}=2 F_{0} / B_{0}$. The voltage $v$ is given by:

$$
v=R i+L \frac{d i}{d t}+N A \frac{d b}{d t}
$$

where $N$ is the number of coil turns, $R$ the coil resistance, $A$ the pole face area and $L$ the coil inductance. Moreover, 
the force $f$ depends on the mass $M$ and the vertical acceleration $\ddot{z}$.

$$
f=M \ddot{z} \quad \text { and } \quad f=K_{b} b
$$

therefore, from (5) and (2) the equation for $\ddot{z}$ is

$$
\ddot{z}=\frac{K_{b} K_{i}}{M} i-\frac{K_{b} K_{\left(z_{t}-z\right)}}{M}\left(z_{t}-z\right)
$$

where $\left(z_{t}-z\right)$ is the air gap between the rail and the electromagnet. Also, from (2) and (6) the current equation is

$$
\frac{d i}{d t}=\frac{V}{L+N A K_{i}}+\frac{N A K_{\left(z_{t}-z\right)}}{L+N A K_{i}}\left(\dot{z}_{t}-\dot{z}\right)-\frac{R i}{L+N A K_{i}}
$$

and from (6) and (7) a state vector can be constructed as follows

$$
X=\left[i \dot{z}\left(z_{t}-z\right)\right]^{T}
$$

with the relevant state space expression given by

$$
\dot{X}=A_{g} X+B_{v} v+B_{z} \dot{z}_{t}, \quad y=C X
$$

where matrices

$$
\begin{aligned}
& A_{g}=\left(\begin{array}{ccc}
-\frac{R}{L+N A K_{i}} & -\frac{N A K_{\left(z_{t}-z\right)}}{L+N A K_{i}} & 0 \\
\frac{K_{b} K_{i}}{M} & 0 & -\frac{K_{b} K_{\left(z_{t}-z\right)}}{M} \\
0 & -1 & 0
\end{array}\right) \\
& \left(\begin{array}{ll}
B_{v} & B_{z}
\end{array}\right)=\left(\begin{array}{cc}
\frac{1}{L+N A K_{i}} & \frac{N A K_{\left(z_{t}-z\right)}}{L+N A K_{i}} \\
0 & 0 \\
0 & 1
\end{array}\right) \\
& C=\left(\begin{array}{ccc}
1 & 0 & 0 \\
K_{i} & 0 & -K_{\left(z_{t}-z\right)} \\
0 & 0 & 1 \\
0 & 1 & 0 \\
\frac{K_{b} K_{i}}{M} & 0 & -\frac{K_{b} K_{\left(z_{t}-z\right)}}{M}
\end{array}\right)
\end{aligned}
$$

Note that the output matrix in (12) refers to all possible measurements that can be considered $\left(y=\left[i, b,\left(z_{t}-\right.\right.\right.$ $\left.z), \dot{z}, \ddot{z}]^{T}\right)$. The parameter values for a one tone suspension system are shown in Table 1 . Note that the maglev system is open-loop unstable.

\begin{tabular}{cc}
\hline$M=1000 \mathrm{~kg}$ & $R=10 \Omega$ \\
$G_{0}=0.015 \mathrm{~m}$ & $L=0.1 \mathrm{H}$ \\
$B_{0}=1 T$ & $N=2000$ \\
$I_{0}=10 A$ & $A=0.01 \mathrm{~m}^{2}$ \\
$F_{0}=10000 \mathrm{~N}$ & \\
\hline
\end{tabular}

Table 1. Parameters of magnetic suspension

\subsection{Inputs}

Two track input characteristics are considered, i.e. deterministic changes such as gradients or curves and stochastic (random) changes in the track position due to misalignments. These are discussed in more details below.
Random input Random behaviour of the rail position is caused as the vehicle moves along by track-laying inaccuracies and steel rail discrepancies. Considering the vertical direction, the velocity variations are quantified by a double-sided power spectrum density (PSD) which in the frequency domain is expressed by

$$
S_{\dot{z}_{t}}=\pi A_{r} V
$$

where, $V$ is the vehicle speed (in this work is taken as $15 \mathrm{~m} / \mathrm{s}$ ) and $A_{r}$ represents the track roughness equal to $1 \times 10^{-7} \mathrm{~m}$ (for a typical high quality track). The corresponding (one-sided) autocorrelation function is given by

$$
R(\tau)=2 \pi^{2} A_{r} V \delta(\tau)
$$

Deterministic input The main deterministic inputs to a suspension for the vertical direction are the transitions onto gradients. In this work, the deterministic input components utilised are shown in Fig. 2 and represent a gradient of $5 \%$ at a vehicle speed of $15 \mathrm{~m} / \mathrm{s}$ and an allowed acceleration of $0.5 \mathrm{~m} / \mathrm{s}^{2}$.

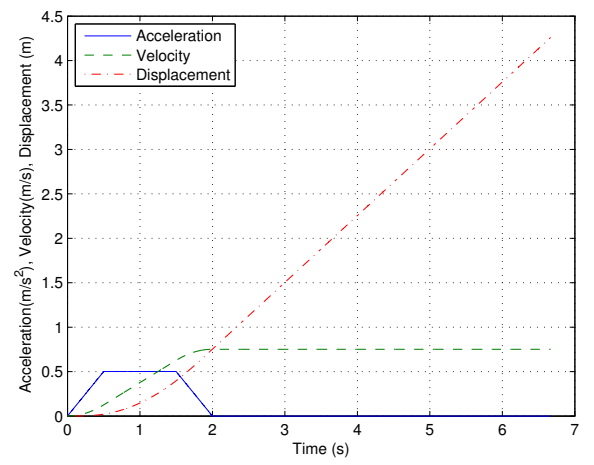

Fig. 2. Deterministic input to the suspension with a vehicle speed of $15 \mathrm{~ms}^{-1}$ and $5 \%$ gradient.

\subsection{Design requirements}

Fundamentally there is a trade off between the deterministic and the stochastic response (ride quality) of the suspension. For slow speed vehicles, performance requirements are described in Goodall (2004) and Goodall (1994). In particular, the practical objective is to minimize both the vertical acceleration (improve ride quality) and the attractive force applied from the electromagnets by minimizing the RMS current variations. These objectives can be can be formally written

$$
\phi_{1}=i_{r m s}, \quad \phi_{2}=\ddot{z}_{r m s}
$$

with the constraints given in Table 2 .

\begin{tabular}{|c|c|}
\hline Constrains & Value \\
\hline RMS acceleration $\left(\simeq 5 \%^{\prime} g^{\prime}\right),\left(\ddot{z}_{r m s}\right)$ & $<0.5 \mathrm{~ms}^{-2}$ \\
RMS gap variation, $\left(\left(z_{t}-z\right)_{r m s}\right)$ & $<5 \mathrm{~mm}$ \\
Air gap deviation (deterministic $),\left(\left(z_{t}-z\right)_{p}\right)$ & $<7.5 \mathrm{~mm}$ \\
Control effort (deterministic),$\left(V_{p}\right)$ & $<300 V\left(3 I_{0} R_{0}\right)$ \\
Settling time, $\left(t_{s}\right)$ & $<3 s$ \\
\hline
\end{tabular}

Table 2. Constrains for the magnetic suspension performance. 


\section{CONTROLLER DESIGN}

Two controller structures are introduced in this section. A classical solution comprising an air gap outer-loop with flux inner-loop is compared with an air gap outer-loop with current inner-loop. A fixed set of classical compensators is considered, namely a proportional plus integral for the inner loops and a phase advance for the outer loop. The aim of the classical solution is twofold first to illustrate the effectiveness of using inner loops for robustness improvement and the difference between the different inner loop measurements; secondly to illustrate the effectiveness of using a genetic algorithm to tune the controller parameters. The tuning procedure is then extended in an LQG framework which is specifically connected to appropriate sensor selection.

\subsection{Classical controller with inner loop design}

Inner loop control is advantageous in controlling a MAGLEV vehicle Goodall (2000). We compare two approaches, a air gap-flux feedback (fig. 3) with the air gapcurrent equivalent. The controller parameters are tuned simultaneously via the evolutionary algorithm NSGAII in an attempt to optimize the control system performance subject to all constraints being satisfied. This design also serves as a baseline for further investigation of schemes with more sensor combinations. The inner loop bandwidth must be within $50 \mathrm{~Hz}-100 \mathrm{~Hz}$ while the outer loop is chosen less than $10 \mathrm{~Hz}$. A phase advance (PA) (16), with $k$ the advance ratio and $\tau$ the time constant, is used to provide adequate phase margin in the range $35^{\circ}-40^{\circ}$.

$$
P I=G_{i} \frac{t_{i} s+1}{t_{i} s} \quad P A=G_{o} \frac{k \tau s+1}{\tau s+1}
$$

The scheme is depicted in Fig.3 for the air gap-flux case. The diagram also applies for the air gap-current case by replacing flux with current measurement.

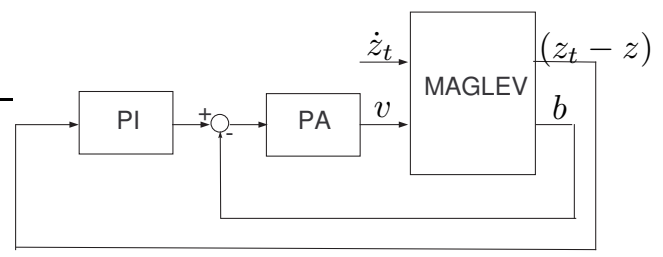

Fig. 3. Classical controller implementation with flux inner loop feedback

\subsection{LQG with sensor optimization}

Linear Quadratic Gaussian control is a well documented in Skogestad (2005) and thus its theoretical details are omitted. Consider the following state space expression

$$
\begin{aligned}
& \dot{x}=A_{g} x+B u+B_{w} \omega_{d} \\
& y=C x+\omega_{n}
\end{aligned}
$$

where, $\omega_{d}$ and $\omega_{n}$ are the process and measurement noises respectively. These are uncorrelated zero-mean Gaussian stochastic processes with constant power spectral densities $W$ and $V$ respectively. In particular, the problem is to find $u=K_{l q g}(s) y$ which minimises the following performance index for every sensor set combination available (this particularly relates to the information provided to the Kalman filter).

$$
J_{L Q G}=E\left\{\lim _{\tau \rightarrow \infty} \frac{1}{\tau} \int_{0}^{\tau}\left[x^{T} Q x+u^{T} R u\right] d t\right\}
$$

Here, $Q$ and $R$ are the state and control weighting functions with $Q=Q^{T} \geq 0$ and $R=R^{T} \geq 0$ of the linear quadratic part of the LQG. Similarly, $W$ and $V$ are the tuning parameters of the Kalman filter part (note that the LQG controller can be designed via the separation principle). The scheme is shown in Fig. 4 with all possible measurements included. For appropriate disturbance rejection, i.e. zero steady state error for the air gap signal, the LQR part is designed on an augmented system with the extra integral state of the air gap (however the Kalman filter is designed on the original state space matrices, but the integral state is later provided by the selector matrix $C_{i}$ ). Note that with five sensors the number of possible combinations to the Kalman filter is $2^{5}-1=31$. It is also

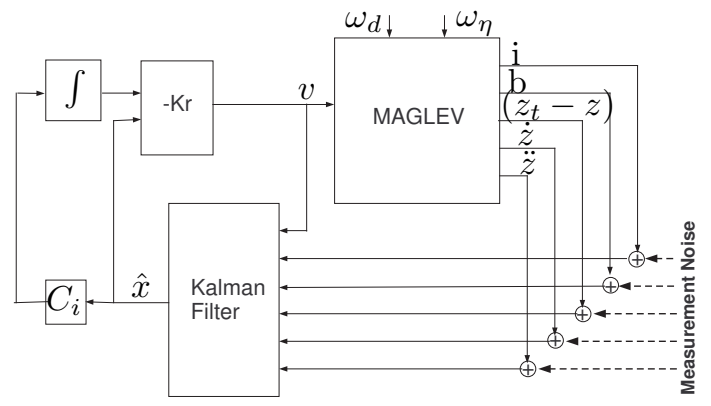

Fig. 4. LQG diagram setup

worth noting that for the LQR design we choose output regulation, i.e. acceleration $(\ddot{z})$, air gap $\left(z_{t}-z\right)$ and the integral of air gap $\left(\int\left(z_{t}-z\right)\right.$ (the last quantity specifically refers to the speed of response). Thus, $Q$ is in fact given by

$$
Q=C_{z}^{T} Q_{z} C_{z}
$$

where $C_{z}$ is the output matrix selecting the above regulated signals, i.e. $\left[\ddot{z}\left(z_{t}-z\right) \quad \int\left(z_{t}-z\right)\right]$ and $Q_{z}$ is the corresponding weight. The Kalman filter is designed such that $E\left\{[x-\hat{x}]^{T}[x-\hat{x}]\right\}$ is minimized.

\section{NSGAII IMPLEMENTATION}

NSGAII is applied in both classical and LQG controller structures, although with different constraints. The parameters used are shown on table 3 . The state feedback tuning requires four variables which are real values. The crossover probability is generally selected to be large in order to have a good mixing of genetic material. The mutation probability is defined as $1 / n_{v}$, where $n_{v}$ is the number of variables. This is appropriate in order to give a mutation probability that mutates an average of one parameter from each individual. For the simulated binary crossover parameter (SBX) and the mutations parameter it was decided to use the default value of 10 and 50 since they provide good distribution of solutions for the algorithm operations. The population size and generation size are set to 50 and 500 


\begin{tabular}{|c|c|}
\hline Parameter & setting \\
\hline Crossover propability & 0.9 \\
Mutation probability & $1 / n_{u}$ \\
SBX parameter & 10 \\
Mutation parameter & 50 \\
Rigid bounds & 1 (yes) \\
Population & 50 \\
Generations & 500 \\
\hline
\end{tabular}

Table 3. NSGA-II Parameters used for the evolution procedure.

respectively for the classical controller optimisation and LQR tuning. For the LQG tuning much less is required, as there is only one variable to tune $(\mathrm{pop}=25, \mathrm{Gen}=5)$. There is no systematic method to define those values as they depend on the nature of the problem. In fact, these values are selected after a few trials. Moreover, the algorithm performance depends on the search space if it's too large the aforementioned generations and population may not be enough. In this work, the search space for both classical and LQG is decided after manually designing an equivalent controller. The penalty function approach Deb (2001) is used to achieve the constraint within limits. The constraint violation for each constraint, $k^{i}$, defined in table 2 is given as in (20).

$$
\omega_{j}\left(k^{i}\right)= \begin{cases}\left|g_{j}\left(k^{i}\right)\right|, & \text { if } g_{j}\left(k^{i}\right)<0 \\ 0 & \text { otherwise }\end{cases}
$$

Each constraint is normalized as in (21) for values less than the predefined and in (22) for values greater than the predefined.

$$
\begin{gathered}
g_{j}=-\frac{k^{i}}{k_{d e s}^{i}}+1 \geq 0 \\
g_{j}=\frac{k^{i}}{k_{d e s}^{i}}-1 \geq 0
\end{gathered}
$$

Where, $k_{\text {des }}^{i}$ is the preset constraint value and $k^{i}$ is the measured value. Equation (22) is not used for LQG design, because all limitations are required to be less than the defined ones. The overall constraint violation is given as in $(23)$.

$$
\Omega\left(k^{(i)}\right)=\sum_{j=1}^{j} \omega_{j}\left(k^{(i)}\right)
$$

This constraint violation is then added to each of the objective functions values ( 24$)$

$$
\Phi_{m}\left(k^{(i)}\right)=\phi_{m}\left(k^{(i)}\right)+R_{m} \Omega\left(k^{(i)}\right)
$$

where $R_{m}$ is the penalty parameter and $\Phi_{m}\left(k^{(i)}\right)$ the objective function value. Remarks on the different controller designs are presented below.

\subsection{Classical controller}

Fig. 5 depicts the pareto-optimality between the ride quality $(\ddot{z})$ and the RMS coil current $\left(i_{r m s}\right)$ for the two controller configurations, i.e the air gap-flux $\left(\left(z_{t}-z\right)-f\right)$ and the air gap-current $\left(\left(z_{z}-z\right)-i\right)$ case. It can be seen that a set of optimized controllers can be chosen which satisfy all constraints for the $\left(z_{t}-z\right)-b$ case but not for the $\left(z_{t}-z\right)-i$ (more complex controller are necessary in the latter case). This can be seen in Table 4 where, both deterministic and stochastic responses are satisfied for all optimum controllers for the $\left(z_{t}-z\right)-b$ case. Robustness to

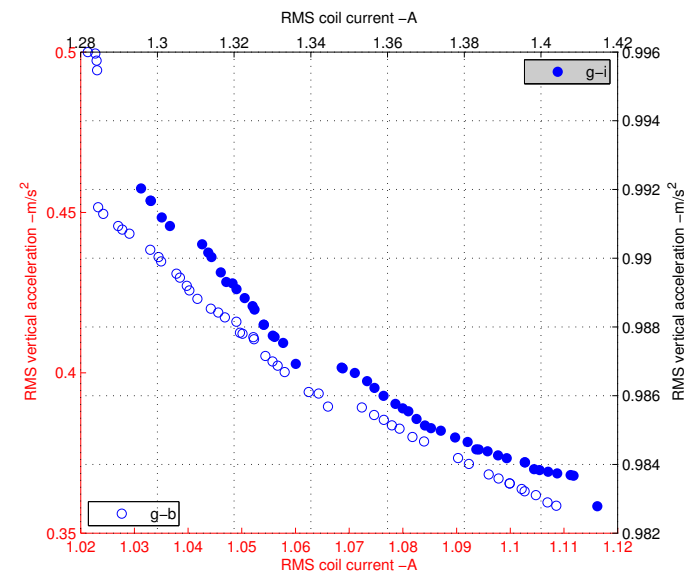

Fig. 5. Optimised controllers for Air gap-flux and Air gapcurrent sensor configurations.

\begin{tabular}{|c|c|c|c|}
\hline & Constrains & $\left(z_{t}-z\right)-b$ & $\left(z_{t}-z\right)-i$ \\
\hline PM(degrees) & $35-45$ & $35-40$ & $\mathbf{6 . 5 - 7}$ \\
$f_{b_{\text {out }}}(H z)$ & $<10$ & $3.2-3.8$ & $\approx 5.8$ \\
$f_{b_{\text {in }}}(H z)$ & $50-100$ & $76-99$ & $\approx 100$ \\
Gap Peak $(\mathrm{mm})$ & $<7.5$ & $\approx 5$ & $\approx 1$ \\
RMS Air Gap $(\mathrm{mm})$ & $<5$ & $\approx 1.5$ & $\approx 1.5$ \\
Control Effort $\left(V_{p}\right)$ & $<300$ & $\approx 10$ & $\approx 30$ \\
RMS $\ddot{z}\left(m^{-2}\right)$ & $<0.5$ & $0.35-0.45$ & $\approx \mathbf{0 . 9 8}$ \\
\hline
\end{tabular}

Table 4. Classical control - constraints values

for each design.

parameter variations is considered only for the $\left(z_{t}-z\right)-b$ configuration since the $\left(z_{t}-z\right)-i$ configuration already violates two of the constraints. A set of optimal controllers, for the extreme cases of $\ddot{z}=0.37 \mathrm{~m} / \mathrm{s}^{-2}$ and $0.45 \mathrm{~m} / \mathrm{s}^{-2}$ is selected, i.e. (25) is the first set of controllers (C1) and (26) is the second set (C2). The mass (M) is varying by $\pm 25 \%$ from the nominal value of $M=1000 \mathrm{~kg}$.

$$
\begin{aligned}
& C 1\left\{P I_{1}=11684 \frac{0.021 s+1}{0.021 s}, P A_{1}=5.5 \frac{0.084 s+1}{0.015 s+1}\{25\right. \\
& C 2\left\{P I_{2}=10063 \frac{0.018 s+1}{0.018 s}, P A_{2}=5.6 \frac{0.115 s+1}{0.016 s+1}\{26\right.
\end{aligned}
$$

The Nichols plot for the minimum, nominal and maximum mass variation is shown in Fig. 6 for the $\mathrm{C} 1$ controller set and it can be seen that the controller can accommodate the mass variations and maintain stability and performance (similarly for the $\mathrm{C} 2$ case). From table 5, can be clearly seen that sufficient robustness properties apply in the case of $\mathrm{C} 1$. The $\mathrm{C} 2$ controller set, being closer to the limits of the constraints, fails to satisfy the ride quality requirement for the case where mass is $750 \mathrm{~kg}$. The disturbance rejection to the mass variation for the first set of controllers is shown in Fig. 7. The disturbance is successfully rejected in less than $3 s$ and the steady state value of the air gap returns to the operating point (note that 0 in the figure refers to the operating condition of $10 \mathrm{~mm}$ ). 


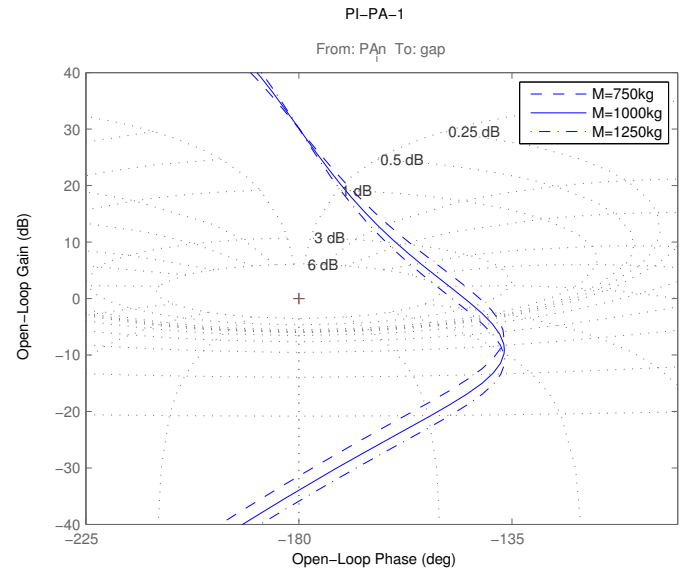

Fig. 6. Mass variation effect on the suspension system for the $\mathrm{C} 1$ controller set.

\begin{tabular}{|c|c|c|c|c|c|c|}
\hline & \multicolumn{2}{|c|}{$\mathrm{M}=750 \mathrm{~kg}$} & \multicolumn{2}{c|}{$\mathrm{M}=1000 \mathrm{~kg}$} & \multicolumn{2}{c|}{$\mathrm{M}=1250 \mathrm{~kg}$} \\
\hline & $\mathrm{C} 1$ & $\mathrm{C} 2$ & $\mathrm{C} 1$ & $\mathrm{C} 2$ & $\mathrm{C} 1$ & $\mathrm{C} 2$ \\
\hline PM (degrees) & 38.2 & 49.9 & 35 & 44.7 & 32 & 42.7 \\
$f_{\text {bout }}(\mathrm{Hz})$ & 4 & 4.9 & 3.2 & 3.8 & 2.8 & 3.22 \\
$f_{\text {bin }}(\mathrm{Hz})$ & 95 & 84 & 95 & 84 & 95 & 84 \\
Gap peak (mm) & 3.6 & 3.4 & 4.9 & 4.6 & 6.3 & 5.9 \\
RMS Gap (mm) & 1.3 & 1.18 & 1.71 & 1.27 & 1.87 & 1.34 \\
RMS $\ddot{z}\left(\mathrm{~ms}^{-2}\right)$ & 0.47 & $\mathbf{0 . 6 1}$ & 0.37 & 0.44 & 0.3 & 0.36 \\
control effort $(\mathrm{V})$ & 25.88 & 24 & 35 & 33 & 45 & 42 \\
\hline
\end{tabular}

Table 5. Constraints values for PI1 and PA1 $0.37 \mathrm{~m} / \mathrm{s}^{2}$.

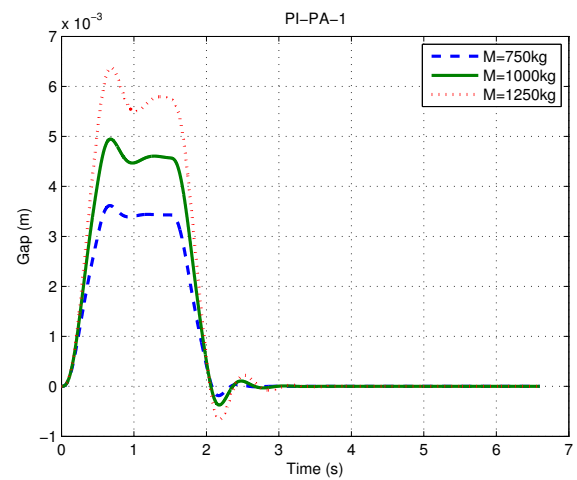

Fig. 7. System response to deterministic input for mass variation of $\pm 25 \%$.

\subsection{Sensor optimization with $L Q G$ controller}

The system is both stabilisable and detectable, and this can be easily found via a modal test. With regards to the separation principle of the LQG design, first the $Q$ and $R$ weights (LQR) are tuned to find the pareto optimality of the objective functions (see fig. 8). Here a small relaxation to the deflection limit is considered (max air gap deflection allowed is $7.3 \mathrm{~mm}$ ) to accommodate the sensor noise effects in the next stage of the Kalman filter design. The above assists in choosing a set of LQR gains, i.e. which has gains of $K_{r_{i, V}}=596 \mathrm{~V} / \mathrm{A}, K_{r_{\dot{z}, V}}=8375 \mathrm{~V} / \mathrm{ms}^{-1}, K_{r_{\left(z_{t}-z\right), V}}=$ $-520992 \mathrm{~V} / \mathrm{m}$. and $\left.K_{\int_{\left(z_{t}-z\right), V}}=-809364 \mathrm{~V} / \mathrm{m}\right]$ that satisfy the design constraints (any can be chosen as far as it is providing the required performance). The subscript

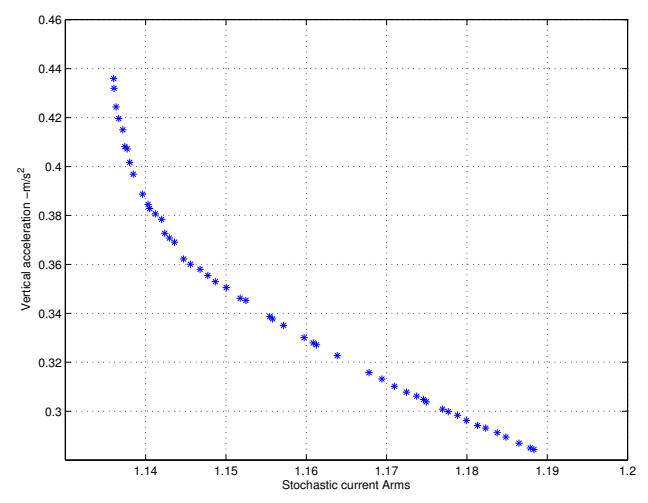

Fig. 8. Pareto optimality with full state feedback

indicates the corresponding state. The next stage is the design of the Kalman filter and the stage where the sensor information becomes critical. In particular the measurement noise weighting is constant and given in (27) for all sensors (this can be found from sensor equipment data sheets or prior simulation of baseline controller designs). In this design the process noise matrix $B_{w}=B_{z}$ and the process noise covariance refers to the track velocity input and is tuned for each sensor set. The objective functions to be minimized are (28) for the deterministic response and (29) for the stochastic response.

$$
\begin{array}{r}
\left.V=\operatorname{diag}\left(V_{i}, V_{b}, V_{\left(z_{t}-z\right)}\right), V_{\dot{z}}, V_{\ddot{z}}\right) \\
\phi_{\text {deterministic }}=\int_{0}^{t}\left|x_{o}-x_{a}\right| d t \\
\phi_{\text {stochastic }}=\operatorname{rms}\left(x_{o}-x_{a}\right)
\end{array}
$$

where, $x_{o}$ are the monitored states of interest of the closed loop with the LQR state feedback (e.g. ideal closed loop) and $x_{a}$ the monitored states of interest of the closed loop with the overall LQG controller (e.g. actual closed loop (prior to adding sensor noise). Note that the sensor information entering the Kalman filter are affected by sensor noise. This makes a total of 6 individual objective functions. Evolution is done in 5 generations with a population of 25 individuals. After the simulations, there are 775 optimum individuals from which to choose the best. The selection procedure of the 'optimum Kalman' estimator is based on the overall penalty parameter (23), which is zero if all constraints are satisfied, and close to zero if the constraints are almost satisfied (see 21). The next criterion is the sum of the objective functions as shown in (30).

$$
S=\sum_{i=1}^{6}\left(\phi_{\text {deterministic }}, \phi_{\text {stochastic }}\right)
$$

Each final population is checked and the individual(s) that give the smallest penalty parameter are selected and among them, the individual that gives the smallest $S$ is selected as the 'optimum Kalman' estimator. This procedure gives an optimum controller for each sensor set combination, with 24 out of 31 sensor set combinations found to meet all constraints. Table 6 illustrates the constraint values for each sensor set with the corresponding 
optimized controller that is selected. The sensor combinations that satisfy all constraints are marked $(\sqrt{ })$. The flux as well as vertical acceleration measurements are good choices satisfying the required constraints. Kalman filter gains with vertical acceleration measurement is $K_{f_{\ddot{z}}}=$ $\left[\begin{array}{lll}-63444 & 2 & -100\end{array}\right]^{T}$ and for the flux measurement is $K_{f_{b}}=\left[\begin{array}{lll}-1268899 & 40 & -2000\end{array}\right]^{T}$. In fact, this can be another criterion for choosing the sensor set with the smallest possible Kalman gains. It is also worth mentioning that adding more sensors increases the fault possibilities on the sensor elements, as well as incorporating more complexity in system implementation A first good option

\begin{tabular}{|c|c|c|c|c|c|c|}
\hline Sensor & $g_{a i r_{r m s}}$ & $g_{a i r_{p}}$ & $V_{p}$ & $\ddot{z}_{r m s}$ & $t_{s}$ & \\
\cline { 2 - 7 } set & $\mathrm{mm}$ & $\mathrm{mm}$ & $\mathrm{V}$ & $m s^{-2}$ & $s$ & \\
\hline $\mathrm{b}$ & 1.4 & 5.3 & 94 & 0.35 & 2.25 & $\sqrt{ }$ \\
$g_{\text {air }}$ & 1.4 & 4.8 & 81 & 0.35 & $\mathbf{6 . 4 3}$ & $\mathrm{x}$ \\
$\ddot{z}$ & 1.4 & 5.3 & 92 & 0.34 & 2.12 & $\sqrt{ }$ \\
$\mathrm{i}, \dot{z}$ & 1.4 & 5.6 & 101 & 0.35 & $\mathbf{6 . 1 7}$ & $\mathrm{x}$ \\
$\mathrm{i}, \ddot{z}$ & 1.4 & 5.3 & 72 & 0.34 & 2.25 & $\sqrt{ }$ \\
$\mathrm{i}, \mathrm{b}, g_{\text {air }}$ & 1.4 & 5.2 & 66 & 0.35 & 2.25 & $\sqrt{ }$ \\
$\mathrm{i}, \mathrm{b}, \dot{z}$ & 1.4 & 5.7 & 70 & 0.35 & 2.3 & $\sqrt{ }$ \\
$\mathrm{i}, g_{\text {air }}, \dot{z}$ & 1.4 & 5.5 & 88 & 0.35 & $\mathbf{6 . 2 2}$ & $\mathrm{x}$ \\
$\mathrm{i}, \mathrm{b}, g_{\text {air }}, \dot{z}$ & 1.4 & 5.6 & 63 & 0.35 & 2.3 & $\sqrt{ }$ \\
$\mathrm{i}, \mathrm{b}, g_{\text {air }}, \ddot{z}$ & 1.4 & 5.3 & 65 & 0.35 & 2.2 & $\sqrt{ }$ \\
$\mathrm{i}, \mathrm{b}, g_{\text {air }}, \dot{z}, \ddot{z}$ & 1.4 & 5.5 & 63 & 0.35 & 2.2 & $\sqrt{ }$ \\
\hline
\end{tabular}

Table 6. Sensor combinations with constraints

$$
\left(g_{\text {air }} \equiv\left(z_{t}-z\right)\right) \text {. }
$$

is the vertical acceleration, with the state estimation from the Kalman filter quite satisfactory as it can be seen from Fig.9. Disturbance rejection is also appropriate in
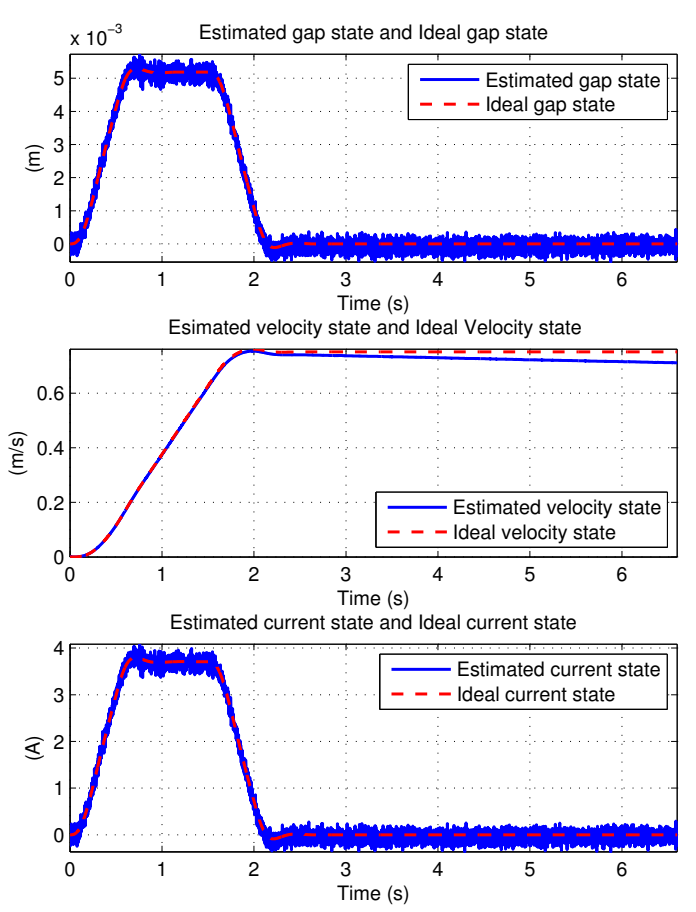

Fig. 9. The state estimates with vertical acceleration measurement.

the deterministic case. The air gap settles to its operating condition (nominal value) within three seconds with small overshoot and undershoot within the predefined limits (note that the Kalman filter is primarily a stochastic estimator thus with smaller Kalman gains a small drift occurs; this can be solved by increasing the Kalman gains however at the expense of larger sensor noise entering the system).

\section{CONCLUSION}

The paper discussed a system study from a sensor optimization point of view for a magnetic suspension system via a heuristic approach (NSGAII) on controller tuning. Two controller cases where presented, a classical case on fixed sensor sets for illustrating the efficacy of the heuristic algorithm on controller tuning. This was extended to an LQG controller design with the particular aim of sensor optimization for the Kalman filter part. The study illustrated that most of sensors sets are able to provide satisfactory control of the magnetic suspension system. Note that the study identifies the minimum sensor sets required for appropriate performance, effectively reducing sensor fault scenarios. In particular, the presented framework aims to identify potential sensor sets that can be used as a basis for future investigation on system fault tolerance via possible controller structure re-scheduling.

\section{ACKNOWLEDGEMENTS}

This work was supported in part under the EPSRC (UK) project Grant Ref. EP/D063965/1 and BAE Systems (SEIC), UK.

\section{REFERENCES}

H.W. Lee, K.C. Kim, and J. Lee. Review of Maglev Train Technologies. IEEE transactions on magnetics, volume 42, pages 1917-1925, July 2006.

K. Deb, A. Pratap, S. Agarwal and T. Meyarivan. A fast and Elitist Multiobjective Genetic Algorithm: NSGAII. IEEE transactions on evolutionary computation, volume 6, No2, pages 182-197, April 2002.

K. Deb Multi-objective Optimization Using evolutionary algorithms. John Wiley $\mathscr{E}$ sons Ltd,2001.

K. Abdullah,D.W. Coit,A.E.Smith Multi-objective optimization using genetic algorithms: A tutorial. Reliability Engineering and systems safety, page992-1007,2006.

P.J.Fleming, R.C.Purshhouse Evolutionary algorithms in control systems engineering:a survey. Control Engineering Practice,page1223-1241, 2002.

R.M.Goodall. Dynamic characteristics in the design of Maglev suspension. IMechE, volume 208, pages 33-41, March 1994 .

R.M.Goodall. Dynamics and control requirements for EMS Maglev suspensions. Proceedings on International Conference on Maglev, volume, pages 926-934, Oct 2004 .

R.M.Goodall On the robustness of flux feedback control for electro-magnetic Maglev controllers. Proc. of $16^{\text {th }}$ Int. Conference on Maglev systems and linear drives, Rio de Janeiro, Brazil, page197-202,June 2000.

R.M.Goodall The theory of electromagnetic levitation. Physics in technology, Vol 16,No5,page207213,September, 1985.

S.Skogestad,I.Postlethwaite. Multivariable feedbak control - Analysis and desig. Wiley 85 Sons., Ltd 2005. 